\title{
Actin and myosin in non-muscle cells
}

\section{Secretion, motility and cell division}

from Robert S. Adelstein

Próteins similar in molecular structure and biological properties to the muscle proteins actin and myosin have been identified in a large number of cells found in both vertebrates and invertebrates. Actin purified from nonmuscle cells has the same monomeric molecular weight as skeletal muscle actin (42,000 daltons), contains a single residue of the unusual amino acid $N^{\top}$ methyl histidine and polymerises into filaments upon alteration of the ionic strength. The details of this polymerisation are not understood but it is thought that actin in non-muscle cells undergoes reversible polymerisationdepolymerisations unlike actin in muscle cells, which is thought to exist only as a filament.

Myosin from vertebrate non-muscle cells resembles muscle myosin (molecular weight 460,000 ) in being composed of heavy chains (200,000 daltons) and light chains $(20,000$ and 16,000 daltons in the case of fibroblasts, platelets and macrophages). Similar to muscle myosin, cytoplasmic myosin possesses an enzymic activity which catalyses the hydrolysis of ATP into ADP and inorganic phosphate. This ATPase activity is markedly enhanced at low jonic strength in the presence of $\mathrm{Mg}^{2+}$ by the addition of actin. It is noteworthy that cytoplasmic myosin interacts with muscle actin and vice versa.

Two major problems occupy the ever expanding number of workers attracted to this field: (1) the location of actin and myosin in non-muscle cells and (2) the function of these proteins (see also Nature, 253; 399; 1975).

Three important cell processes have been postulated to involve cytoplasmic actin and myosin: secretion, cell division, and cell motility. It is important to note that all the evidence presented to date is at best circumstantial, in that no direct evidence for the role of actin and myosin in any of these processes has been reported.

In a recent article Burridge and Phillips (Nature, 254, 526; 1975) presented evidence for the association of actin with the secretory granules of the adrenal medulla. These granules contain neurotransmitters which are released, following exocytosis into the blood stream. The authors succeeded in preparing membranes from partially lysed chromaffin granules which bound rabbit skeletal muscle actin (and very small quantities of chicken smooth muscle myosin). Binding was indicated by finding the contractile proteins and lysed membranes in the same fraction following sedimentation in a sucrose gradient.

The authors supplemented these studies with electron micrographs showing lysed chromaffin granule membranes associated with rabbit skeletal muscle actin filaments (the original photographs being superior in quality to those reproduced in Nature). In muscle cells actin is uniquely attached to a Z-line so that 'decoration' of the actin filament with a fragment of myosin, heavy menomyosin, leads to the formation of polarised 'arrowheads' pointing away from the Z-line attachment site. Unfortunately this uniform polarity is lacking in the preparation of membranes and actin filaments examined by these authors raising the possibility that at least some of the filaments might not be uniquely attached to the membrane.

Puszkin and Kochwa in a preliminary communication (J. biol. Chem., 249,7711 ; 1974) presented data suggesting that contractile proteins are important in the release of neurotransmitters from brain tissue. They isolated a complex of actin and 'relaxing proteins' from rat brain synaptosomal membranes. The actin-'relaxing proteins' complex interacted with symaptic vesicles from brain, preloaded with ${ }^{14} \mathrm{C}$ glutamate and presumably containing brain myosin. The results of the interaction (in the presence of $\mathrm{Ca}^{2+}$ ) were release of the labelled glutamate and activation of the Mg-ATPase activity of the myosin. The work is incomplete in that neither the relaxing proteins (presumably troponin) nor the myosin were positively identified. Moreover the release of ${ }^{14} \mathrm{C}$-glutamate is assumed to correlate with the secretion of neurotransmitter.

Evidence that actin in HeLa cells (human tumour cells grown in tissue culture) might play a part during one phase of cell division was first proferred by Schroeder (Proc. natn. Acad. Sci. U.S.A., 70, 1688; 1973) who identified actin in the contractile ring of these cells. The identification was made by treating the cells with glycerine so they would be permeable to heavy meromyosin which interacted with actin filaments to form the unique arrowhead pattern alluded to above. Again, the evidence is circumstantial. The presence of actin implies, but does not prove, that it is functioning (perhaps with myosin?) to cleave the cells.

A role for actin in cell motility and phagocytosis was suggested by Boxer et al. (New Engl. J. Med., 291, 1093;
1974) who investigated polymorphonuclear leukocytes isolated from a patient suffering multiple infections. These cells failed to migrate normally an ingested particles at a slower rate than controls. Actin isolated from these cells could not polymerise to the same extent as actin from the white cells of normal controls. It must be stressed that only a single patient's cells were examined and that the process of actin polymerisation using nonmuscle actin is not well understood at present. But the principle of using a cell showing a defect in its motile behaviour as a source for studying actin is important.

Future experiments in this field should be directed at: (1) complete characterisation of the various proteins and their interaction; (2) the in situ identification of the contractile proteins using antibodies that have been shown to be specific for either actin or myosin by a number of rigorous criteria; (3) the removal of a specific link (for example, $\mathrm{Ca}^{2+}$ ATP, troponin) in the contractile mechanism which results in changes that can be related to cell function; (4) further studies looking for specific defects in the contractile proteins of abnormally functioning cells; (5) identification of a specific inhibitor of actin-myosin interaction which can be used in vivo (as well as in vitro) to study changes in cell function. The list, of course, can be ex-

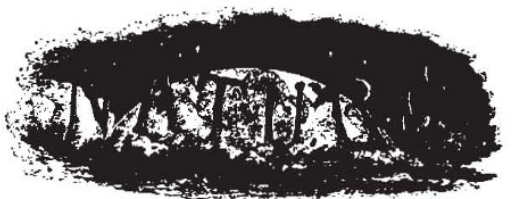

\section{A hundred years ago}

Curious Phenomenon of Light

RowING on Loch Lomond recently, above Luss, there were seen to the north-west, at an apparent distance of about 100 yards, two bright lines of prismatic light, $60^{\circ}$ apart and on the level of the water. Their length seemed to equal the breadth of a rainbow. Their violet ends were towards each other, and were joined by a line of dull white light, to the middle of which the sun and the spectator were at right angles. Standing in the boat, the colour and brilliancy were lost, and only a diffuse white light was visible. The time was 10 A.M. The sun was hot, the sky cloudless, the air hazy and still, and the toch a mirror. This apparition fled before our approach for some minutes, till dispelled by a slight breeze, which rippled the water.

Luss

WM. M'LAurin

from Nature, 12, 26; May 13, 1875 